\title{
Psicologia, espiritualidade/religiosidade e cuidados paliativos: uma revisão integrativa
}

\author{
Psychology, spituality/religiosity and \\ palliative care: an integrative review
}

Beatriz Fonseca Aguiar ${ }^{1}$ (1)
Jéssica Plácido Silva ${ }^{2}$ (1)

'Escola Bahiana de Medicina e Saúde Pública (Salvador). Bahia, Brasil. beatrizaguiarpsi@hotmail.com ${ }^{2}$ Autora para correspondência. Escola Bahiana de Medicina e Saúde Pública (Salvador), Universidade Federal da Bahia (Salvador). Bahia, Brasil. jelplacido@hotmail.com

RESUMO | INTRODUÇÃO: A espiritualidade faz parte da constituição psíquica, gerando comportamentos que influenciam na percepção da dicotomia saúde/doença e para pacientes em cuidados paliativos é considerada uma necessidade mais urgente por conta do momento de vulnerabilidade. OBJETIVO: Analisar na literatura produções sobre as interfaces entre psicologia e espiritualidade/religiosidade no contexto de cuidados paliativos. MÉTODO: Revisão Integrativa da literatura, com coleta de dados no mês de fevereiro de 2018, nas bases de dados PEPSIC, SCIELO, SCOPUS, PubMed, de artigos publicados entre janeiro de 2015 e dezembro de 2018 e os descritores utilizados foram "psyholog*", "spirit*" e "palliative care". RESULTADOS E DISCUSSÃO: Foram incluídos 05 artigos, em que a espiritualidade aparece como uma necessidade do paciente e de sua família, fazendo parte do cuidado integral fornecido/outorgado ao paciente em cuidados paliativos. Os conceitos de espiritualidade apresentados são convergentes, existem práticas e abordagens que possibilitam à psicóloga trabalhar a dimensão espiritual nos cuidados paliativos. CONSIDERAÇõES FINAIS: É relevante considerar a dimensão espiritual de pacientes em contexto de cuidados paliativos, portanto, faz-se necessário essa temática durante a formação acadêmica em Psicologia, bem como o desenvolvimento de novos estudos realizados sobre esse tema por psicólogos.

PALAVRAS-CHAVE: Cuidados paliativos. Espiritualidade. Psicologia.

\begin{abstract}
INTRODUCTION: The spirituality is part of the psychic constitution, generating behaviors that influence the perception of the health / disease dichotomy and for patients in palliative care is considered a more urgent need due to the moment of vulnerability. OBJECTIVE: to understand how the spiritual dimension is approached in the context of palliative care. METHOD: Integrative Review of the literature, with data collection in February 2018, in the databases PEPSIC, SCIELO, SCOPUS, PubMed, of articles published between January 2015 and December 2018 and the descriptors used were "psyholog *", "Spirit *" and "palliative care". RESULTS AND DISCUSSION: 05 articles were included, in which spirituality appears as a necessity of the patient and his family, being part of the integral care provided / granted to the patient in palliative care. The concepts of spirituality presented are convergent, there are practices and approaches that allow the psychologist to work the spiritual dimension in palliative care. FINAL CONSIDERATIONS: It is relevant to consider the spiritual dimension of patients in the context of palliative care, therefore, it is necessary this theme during the academic training in Psychology, as well as the development of new studies carried out on this topic by psychologists.
\end{abstract}

KEYWORDS: Palliative care. Spirituality. Psychology. 


\section{Introdução}

A "religião" é o sistema organizado de práticas e crenças, incluindo ritos e símbolos, que é compartilhado entre as pessoas do mesmo grupo religioso com o fim de aproximar as pessoas do transcendente, sendo a "religiosidade" a "prática institucionalizada da religião" (Koenig, King, \& Carson, 2012). Já a espiritualidade "está mais voltada para o eu e o transcendente, práticas intrínsecas onde o indivíduo sozinho busca, através da religião ou não, significados para a vida e para Deus" (Koenig, King, \& Carson, 2012; Zerbetto et al, 2017). Embora os conceitos apresentem aproximações e sejam trabalhados como um único construto na literatura de uma forma geral, é necessário fazer essa diferenciação entre ambos, pois uma pessoa que não segue uma religião pode buscar a espiritualidade, assim como, uma pessoa com religião não necessariamente vai ter interesse em se conectar com o transcendente (Nascimento et al., 2013).

A presença da Religião e Espiritualidade (R/E) é muito marcante em toda história da humanidade (Henning \& Geronasso, 2009). Nos registros antigos de História e Arqueologia existem relatos e estudos que mostram como a religião, por séculos, serviu de diretriz ética e moral, esculpindo posturas e atitudes ideais para os sujeitos, reduzindo comportamentos nocivos contra si e contra o outro (Peters, 2015). A religião e a crença religiosa são elementos culturais importantes por influenciarem e guiarem a forma que um indivíduo percebe situações e se comporta na sociedade (Peres, Simão, \& Nasello, 2007).

Nesse sentido, como a R/E são muito relevantes para o sujeito, elas influenciam no cuidado e na evolução dos problemas de saúde dos pacientes (MoreiraAlmeida, 2009). Muitos estudos mostram a R/E estão relacionadas com indicadores de uma melhor qualidade de vida, resultando no impacto benéfico do envolvimento religioso (Moreira-Almeida, 2009), como por exemplo a redução do estresse, na saúde entre indivíduos com alguma deficiência e/ou alguma doença clínica (Moreira-Almeida et al., 2006). Além disso, pessoas que estão inseridas em um grupo religioso possuem menor probabilidade de cometer suicídio, usar drogas abusivamente (lícitas e ilícitas) e menor índice de depressão (Borges et al., 2015).
Apesar da importância que a religião e a espiritualidade possuem na cultura e para a população, nos cursos de saúde, em geral, existe pouca preparação que qualifique os profissionais dessa área a lidar com questões religiosas do paciente em sua prática (Moreira-Almeida, (2009); Oliveira \& Junges, 2012; Marques, Marques, \& Ribeiro, 2016). Quando o profissional de saúde é psicólogo, a situação fica ainda mais difícil, pois durante muito tempo, foi exigido da profissional uma certa neutralidade diante da presença da R/E apresentada por um paciente.

Durante muito tempo ciência e espiritualidade/religiosidade não podiam caminhar juntas, uma vez que eram vistas como incompatíveis e divergentes. Destarte, a Psicologia, para se firmar enquanto ciência psicológica, precisou se distanciar de temas que envolvessem religião e R/E, estando estas até relacionados a psicopatologias. Todavia, tais construtos tem sido analisados como um fatores substanciais para atribuição de sentido e melhor qualidade de vida (Melo et al., 2015). Para além disto, a crise existencial da pós-modernidade contribuiu para a busca de amparo e valores dentro da R/E (Oliveira \& Junges, 2012).

Desta forma, é dever do psicólogo considerar esses fatores como relevantes de serem trabalhados em contexto terapêutico, visto que fazem parte da constituição psíquica e, consequentemente, geram comportamentos e crenças que influenciam na forma como pacientes e profissionais percebem a díade saúde/ doença (Henning \& Geronasso, 2009; Borges, Santos, \& Pinheiro, 2015). Á vista disso, compreende-se a recente ascensão da temática da R/E nos espaços acadêmicos psicológicos, assim como um reconhecimento das dificuldades por parte da área da psicologia para lidar com as questões religiosas e espirituais dos indivíduos e a ausência, em números e conteúdo, de estudos realizados (Moreira-Almeida, 2009).

Um estudo feito por Henning-Geronasso \& Moré (2015) com dez psicólogos se propôs a apontar quais os manejos que são desenvolvidos no contexto terapêutico em relação ao surgimento de demandas relacionadas à dimensão religiosa/espiritual. Compreendeu-se que o profissional da psicologia não deve confrontar com a R/E do paciente e nem permitir que seus próprios valores religiosos/ espirituais intervenham no processo terapêutico. 
Além disso, o estudo relatou a possibilidade de valerse de recursos aportados na R/E dos pacientes.

No contexto de cuidados paliativos, em que o psicólogo faz parte da equipe multiprofissional, a espiritualidade é considerada uma necessidade mais urgente para o paciente por conta do momento de vulnerabilidade, tanto física quanto psíquica, com a iminência da morte (Alves, Melo, Andrade, \& Sousa, 2014). Os cuidados paliativos podem ser definidos como cuidados gerais e direcionados à indivíduos cuja a enfermidade não responde mais a tratamentos médicos, com o objetivo de promover uma melhor qualidade de vida para o paciente e para a família, diminuição da dor, combate de sintomas estressores e angustiantes, questões físicas, psicossociais e espirituais, e também contribui para ajudar o indivíduo a elaborar mecanismos de enfretamento diante do prognóstico terminal (Alves et al., 2014; Evangelista, Lopes, Costa, Abrão, et al., 2016; Gomes et al., 2016).

Para pacientes em cuidados paliativos, a espiritualidade proporciona maior conforto, alívio de sintoma físico, auxílio no tratamento médico convencional e ajuda a elaborar um sentido para a tríade dor, culpa e morte, além de um maior controle emocional diante da finitude da vida (Arrieira et al., 2018). Cervelin \& Kruse, (2014) realizaram um estudo onde foi possível observar através da análise de livros-textos sobre cuidados paliativos que alguns indivíduos conseguem achar na religião suporte para enfrentar a dor e o sofrimento causados pela terminalidade da doença. Nesse mesmo estudo, as autoras encontraram também que a R/E proporcionam uma melhor qualidade de vida para os pacientes, traduzindo assim a existência de uma relação positiva entre esses fenômenos.

O estudo com psicólogos realizado por Alves, Melo, Andrade \& Souza (2014) se propôs a estudar os saberes e as práticas sobre os cuidados paliativos para esses profissionais. Foi encontrado que os psicólogos entrevistados direcionavam suas práticas a partir de três eixos. O primeiro diz respeito a técnicas que a psicologia oferece, como apoio psicológico com o objetivo de elaborar aspectos emocionais, a escuta qualificada e a psicoeducação, tanto para o paciente, como para a família e a equipe multiprofissional de saúde. O segundo eixo diz respeito aos aspectos assistenciais, principalmente frente à família, oferecendo suporte para que esta saiba como proceder da melhor forma na melhoria da qualidade de vida de seu ente que está adoecido. O terceiro eixo diz respeito aos aspectos relacionados ao fator espiritual e ao morrer, que podem contribuir para uma melhoria da qualidade de vida e até proporcionar uma "boa morte" (Alves et al., 2014).

Nesse sentido, o presente trabalho tem como objetivo geral analisar na literatura produções sobre as interfaces entre psicologia e espiritualidade/religiosidade no contexto de cuidados paliativos, através de uma revisão integrativa, tendo em vista a deficiência da relação entre R/E e as práticas da Psicologia nos cursos de graduação. Os objetivos específicos são: descrever as definições de espiritualidade, religiosidade e religião; identificar a importância em abordar a espiritualidade em cuidados paliativos e identificar técnicas e abordagens para trabalhar a espiritualidade em cuidados paliativos.

\section{Método de pesquisa}

Trata-se de uma revisão integrativa da literatura. Este tipo de revisão é um método amplo que proporciona uma compreensão holística do objeto que está sendo estudado e possibilita também a análise de estudos prévios e relevantes (Mendes et al., 2008). Ademais, permite a utilização de pesquisas com abordagens quantitativas e qualitativas, além da literatura teórica e a empírica, e também o conhecimento síntese de múltiplos estudos acerca de um determinado assunto. (Mendes et al., 2008; Pompeo et al., 2009).

O tema definido para este trabalho foi "cuidados paliativos, espiritualidade e psicologia". As perguntas de pesquisa foram: "quais as interfaces entre espiritualidade/religião e psicologia no contexto de cuidados paliativos? Existem diretrizes práticas para a atuação do psicóloga na área?" A partir das questões de pesquisa identificam-se os descritores spirit* $A N D$ psycholog* AND "palliative care". Os critérios de inclusão foram: artigos publicados entre 2015 e 2018, artigos escritos por psicólogos, ou que tratem sobre a prática dos psicólogos, que abordem sobre a relação da espiritualidade e cuidados paliativos. Os critérios de exclusão foram: artigos fechados; artigos duplicados; artigos escritos por outros profissionais e sobre outras áreas de saúde. 
As buscas de pesquisa foram realizadas nos meses de fevereiro e março de 2019, nas plataformas Scientific Electronic Library Online (SciELO), Pubmed, Scopus e Periódicos Eletrônicos de Psicologia (PePSIC). Foram identificados no total 63 artigos publicados entre janeiro de 2015 a dezembro de 2018. Após a aplicação dos critérios de inclusão e exclusão, 5 artigos foram selecionados para compor o estudo, já que a maioria dos artigos encontrados eram da área de medicina ou enfermagem. Tal resultado evidencia a pouca quantidade de literatura nesta interface específica entre psicologia, espiritualidade/religiosidade e cuidados paliativos.

Após a identificação e seleção dos artigos, a amostra foi caracterizada segundo os títulos dos artigos; os autores; os anos de publicações; os locais em que os artigos foram publicados; as revistas em que foram publicados; os objetivos; os métodos utilizados para a realização da pesquisa. Em seguida, os artigos foram analisados segundo as seguintes categorias: (I) os conceitos de espiritualidade, religiosidade e religião; (II) a importância em abordar a espiritualidade em contexto de cuidados paliativos; (III) as técnicas e abordagens apresentadas para trabalhar a espiritualidade nos cuidados paliativos; (IV) as dificuldades e limitações. Por fim, foi realizado a síntese do conhecimento identificado nos estudos e a conclusão.

\section{Resultados e discussão}

\section{Caracterização da amostra}

Foram encontrados 63 artigos na busca conforme os descritores nas bases de dados citadas. Após aplicados os critérios de exclusão e inclusão, 05 artigos mostraram-se aptos à análise. A Tabela 01 oferece a caracterização da amostra.

Tabela 1. Dados comparativos dos artigos em análise: título, autores e ano de publicação, local de execução da pesquisa, revista em que o estudo foi publicado e o método utilizado

\begin{tabular}{|c|c|c|c|c|c|c|}
\hline Art. & Título & Ano & Local & Autores & Revistas & Método \\
\hline 01 & $\begin{array}{l}\text { A Espiritualidade como } \\
\text { Estratégia de } \\
\text { Enfrentamento para } \\
\text { Familiares de Pacientes } \\
\text { adultos em Cuidados } \\
\text { paliativos }\end{array}$ & 2017 & $\begin{array}{l}\text { Rio de } \\
\text { Janeiro }\end{array}$ & $\begin{array}{l}\text { Barbosa, } \\
\text { Ferreira, } \\
\text { Melo e } \\
\text { Costa }\end{array}$ & $\begin{array}{l}\text { Revista Sociedade } \\
\text { Brasileira de } \\
\text { Psicologia } \\
\text { Hospitalar }\end{array}$ & $\begin{array}{c}\text { Estudo } \\
\text { Qualitativo }\end{array}$ \\
\hline 02 & $\begin{array}{l}\text { Clinical Hypnosis, } \\
\text { Mindfulness and } \\
\text { Spirituality in Palliative } \\
\text { Care }\end{array}$ & 2018 & Milão & Casula & $\begin{array}{l}\text { Annals of } \\
\text { Palliative } \\
\text { Medicine }\end{array}$ & $\begin{array}{l}\text { Artigo de } \\
\text { Revisão }\end{array}$ \\
\hline 03 & $\begin{array}{c}\text { Espiritualidade e } \\
\text { Terapia Ocupacional: } \\
\text { Reflexões em Cuidados } \\
\text { Paliativos }\end{array}$ & 2015 & Belém & $\begin{array}{c}\text { Elmescany e } \\
\text { Barros }\end{array}$ & $\begin{array}{l}\text { Revista Nufen: } \\
\text { Phenomenology } \\
\text { and } \\
\text { interdisciplinarity }\end{array}$ & $\begin{array}{c}\text { Pesquisa } \\
\text { Bibliográfica }\end{array}$ \\
\hline 04 & $\begin{array}{c}\text { Significados da } \\
\text { Espiritualidade para } \\
\text { دacientes com Câncer em } \\
\text { Cuidados Paliativos }\end{array}$ & 2017 & Campinas & $\begin{array}{c}\text { Benites, Neme, } \\
\text { e Santos }\end{array}$ & $\begin{array}{l}\text { Estudos de } \\
\text { Psicologia }\end{array}$ & $\begin{array}{c}\text { Pesquisa } \\
\text { Qualitativa }\end{array}$ \\
\hline 05 & $\begin{array}{l}\text { Spirituality in young } \\
\text { adults with end-stage }\end{array}$ & 2017 & $\begin{array}{l}\text { Washington, } \\
\text { DC }\end{array}$ & Mistretta & $\begin{array}{l}\text { Annals of } \\
\text { Palliative } \\
\text { Medicine }\end{array}$ & $\begin{array}{l}\text { Artigo de } \\
\text { Periódico }\end{array}$ \\
\hline
\end{tabular}


Com referência no objetivo deste estudo, os objetivos gerais dos artigos 01 e 04 se propuseram a estudar o significado e expressão da espiritualidade para familiares e para pacientes, respectivamente. $\mathrm{O}$ artigo 02 buscou a expressão da integração dos aspectos psicológicos e espirituais através da hipnose clínica e do mindfulness. 0 artigo 03 pretendeu discutir a espiritualidade em cuidados paliativos na prática da terapia ocupacional. Apesar de todos os objetivos tratarem sobre a espiritualidade, o artigo 05 teve objetivo mais singular, onde pretendeu analisar de forma geral o conhecimento acerca da espiritualidade em paciente jovens adultos em cuidados paliativos oncológicos.

\section{Conceito de espiritualidade, religião e religiosidade}

O conceito de espiritualidade foi apresentado em todos os cinco artigos. O conceito de religião apareceu nos artigos 01, 03, 04 e 05. O conceito de religiosidade apareceu apenas no artigo 04.

Os artigos apresentaram definições similares sobre o conceito da espiritualidade, embora de autores diferentes. Através da análise de cada conceito apresentado em cada artigo, foi possível criar treze ideias distintas, apresentados na tabela 2 a seguir:

Tabela 2. Principais ideias do conceito de Espiritualidade apresentadas nos artigos com a referência original citada no artigo

\begin{tabular}{|c|c|}
\hline Ideias & Artigo/Autor(es) citado(s) \\
\hline $\begin{array}{l}\text { Aproximação/Conexão com o } \\
\text { transcendente }\end{array}$ & $\begin{array}{l}\text { Artigo 01/Silva, } 2011 \\
\text { Artigo 02/Yapko, 2011; Refuge, 2013; Paal, Helo \& Frick, } 2015 \\
\text { Artigo 03/Billock, 2002; Bifulco, 2006; Saporetti, } 2009\end{array}$ \\
\hline $\begin{array}{l}\text { Transcende a existência } \\
\text { humana }\end{array}$ & $\begin{array}{l}\text { Artigo 01/ Pessini \& Bertachini, } 2010 \\
\text { Artigo 03/Bifulco, } 2006\end{array}$ \\
\hline Experiência individual & $\begin{array}{l}\text { Artigo 01/Silva, } 2011 \\
\text { Artigo 04/Saad, Masieiro \& Battistella, 2001; Guimarães \& Azevum, } \\
2007\end{array}$ \\
\hline $\begin{array}{l}\text { Está relacionada com o sentido } \\
\text { da vida }\end{array}$ & $\begin{array}{l}\text { Artigo 01/ Pessini \& Bertachini, 2010; Silva, } 2011 \\
\text { Artigo 03/Bifulco, 2006; Saporetti, 2009 } \\
\text { Artigo 04/Saad, Masieiro \& Battistella, 2001; Guimarães \& Azevum, } \\
\text { 2007; Kovacs, } 2007\end{array}$ \\
\hline $\begin{array}{l}\text { Experiência do encontro com } \\
\text { Deus }\end{array}$ & Artigo 01/ Pessini \& Bertachini, 2010 \\
\hline $\begin{array}{l}\text { Pode estar ou não associada a } \\
\text { uma religião }\end{array}$ & $\begin{array}{l}\text { Artigo 02/Yapko, 2011; Refuge, 2013; Paal, Helo \& Frick, } 2015 \\
\text { Artigo 04/Saad, Masieiro \& Battistella, 2001; Guimarães \& Azevum, } \\
2007\end{array}$ \\
\hline Conexão com a natureza & Artigo 02/Yapko, 2011; Refuge, 2013; Paal, Helo \& Frick, 2015 \\
\hline Autotranscendência & $\begin{array}{l}\text { Artigo 03/Pessini \& Bertachini, } 2010 \\
\text { Artigo 05/Benson, Rochlkepartain \& Rude, } 2003\end{array}$ \\
\hline $\begin{array}{l}\text { Conexão e busca por "algo } \\
\text { maior" }\end{array}$ & $\begin{array}{l}\text { Artigo 02/Yapko, 2011; Refuge, 2013; Paal, Helo \& Frick, } 2015 \\
\text { Artigo 05/Benson, Rochlkepartain \& Rude, } 2003\end{array}$ \\
\hline $\begin{array}{l}\text { Envolve a existência humana } \\
\text { em todas as dimensões }\end{array}$ & Artigo 03/Billock, 2002; Saporetti, 2009; Pessini \& Bertachini, 2010 \\
\hline Fenômeno interno e metafísico & Artigo 03/Billock, 2002 \\
\hline É universal & Artigo 04/Guimarães \& Azevum, 2007 \\
\hline $\begin{array}{l}\text { Elemento inerente ao ser } \\
\text { humano }\end{array}$ & Artigo 04/Valle, 2005 \\
\hline
\end{tabular}


Apesar de o conceito de espiritualidade não ser universal, pôde-se perceber que os conceitos analisados nos artigos se assemelham e se aproximam. As ideias que mais apareceram (presentes em três artigos) foram a busca do sentido de vida e a aproximação/ conexão com o transcendente. Segundo Barbosa, Ferreira, Melo, \& Costa, (2017), o indivíduo em cuidado paliativo possui uma necessidade por respostas, principalmente sobre o real significado de sua existência no momento em que se confronta com seu processo de adoecimento e sua finitude. Quanto a busca/conexão com o transcendente, proporciona aos paciente a atribuição de sentido para a sua própria morte, servindo também como suporte de enfrentamento para a família diante da perda eminente de um ente querido (Arrieira et al., 2018) .

De uma forma geral, a área emergente da R/E e saúde tem utilizado o pesquisador Koenig como referência conceitual (Koenig, 2004; Koenig, Hooten, LindsayCalkins, \& Meador, 2010; Koenig, 2012) e em nenhum dos artigos da presente pesquisa citam o autor quando conceituam R/E. Apesar disso acontecer, as ideias conceituais apresentadas tanto para religião, quanto para espiritualidade convergem aos conceitos elaborados por Koenig.

No que se refere ao conceito de religiosidade, o artigo 04 foi o único estudo a definiu, como sendo uma "experiência religiosa" que faz parte da esfera espiritual e pode ser uma das formas de expressar a mesma (Benites et al., 2017). Pode-se perceber nos estudos uma lacuna, não só sobre a definição da religiosidade, bem como a expressão e relevância desse aspecto para pacientes em contexto de cuidados paliativos e seus familiares.

Referente ao conceito de religião, presente em quatro dos cinco artigos analisados, pôde-se perceber uma coesão quanto as definições. De uma forma geral, nos artigos 01, 04 e 05 a religião apareceu como um construto relacionado a um conjunto de práticas, rituais e símbolos de um determinado grupo religioso e faz parte da espiritualidade. $\mathrm{O}$ artigo 03 apresentou uma conceituação etimológica da religião., descrevendo que "a palavra religião vem do latim religio, na qual a raiz 're' (novamente) e 'ligare' (conectar)", ou seja, a busca pela reconexão com o algo/ser superior (Elmescany \& Barros, 2015).

\section{A importância em abordar a espiritualidade em cuidados paliativos}

Um estudo realizado por Arrieira et al. (2018) com paciente em cuidados paliativos e profissionais da equipe interdisciplinar que efetivaram esse cuidado, mostrou que existe uma demanda espiritual por parte dos pacientes que não pode ser ignorada pela equipe e esse cuidado espiritual exercido pelos profissionais impactam positivamente e fortalecem a confiança entre a tríade equipe-paciente-família.

Quatro dos artigos analisados apresentam em seu conteúdo a importância da abordagem da espiritualidade em cuidados paliativos (01, 03, 04 e 05). Os resultados encontrados convergiram bastante. Esses artigos evidenciaram, de uma forma geral, o reconhecimento da importância do aspecto espiritual do paciente e da família dentro do cuidado paliativo para a manutenção da esperança e da fé, para amenizar as angústias causadas ao se confrontar com a própria finitude, para a atribuição de sentido à vida.

Logo, observou-se através da análise dessas publicações que a espiritualidade integra o conjunto de práticas dos cuidados paliativos por ser uma necessidade advinda do paciente e de sua família. Além disso, considerar a espiritualidade nesse contexto possibilita uma melhor compreensão, tanto por parte do sujeito quanto por parte do profissional, do processo relacionado a doença terminal vivenciada, bem como um conforto para questões relacionadas ao sentido da vida e também contribui para fortificar a aliança entre paciente/equipe e família/equipe (Elmescany \& Barros, 2015; Barbosa et al., 2017; Benites et al., 2017; Mistretta, 2017).

O paciente deseja ser tratado como um todo, como uma pessoa dotada de aspectos biológicos, sociais, emocionais e espirituais (Okon, 2005 apud Peres, Arantes, Lessa, \& Caous, 2007), e a não abordagem de qualquer um desses aspectos nos cuidados em saúde implica na incompletude do tratamento. No contexto de cuidados paliativos os pacientes estão em fim de vida e a transcendência da existência passa a ser muito significativa nesse momento, sendo assim, transcender é buscar significado, e a espiritualidade é o caminho (Peres et al., 2007). 
Técnicas e abordagens para trabalhar a espiritualidade em cuidados paliativos

A espiritualidade tem ganhado cada vez mais destaque dentro dos cuidados direcionados a pacientes em estado terminal (Benites et al., 2017). No entanto é preciso compreender como os profissionais de saúde agem ao se deparar com as demandas religiosas e espirituais do outro. Nos artigos analisados foram apresentadas algumas técnicas e abordagens que facilitam o manejo para trabalhar aspectos espirituais do paciente e da família.

O artigo 01 apresenta não só a importância de reconhecer e trabalhar a espiritualidade dos pacientes e seus familiares, mas também a necessidade de competência e aptidão que o profissional de saúde deve possuir. $O$ enfrentamento religioso pode ser identificado como uma fonte para alívio de angústia. O artigo traz também, de forma breve, a "escuta qualificada, estar presente, prover esperança" (Barbosa et al., 2017) como intervenções possíveis para a manutenção da espiritualidade de forma positiva do outro.

O artigo 02 expõe a hipnose clínica e o mindfulness como duas técnicas possíveis para se trabalhar com pacientes em cuidados paliativos que possuem demandas espirituais. A hipnose é conceituada nesse artigo como um conjunto de "fenômenos caracterizados por respostas a sugestões de modulação de sensações, imagens, comportamentos, afetos e significado da experiência em que o paciente vive no momento" (Casula, 2018). O artigo apresenta a prática da hipnose clínica como facilitadora da descoberta e fortalecimento de recursos internos do paciente, para que este compreenda que não está sozinho e sinta seu medo e sua angustia esvair. A clínica da hipnose é realizada através de sugestões com diretrizes feitas pelo hipnoterapeuta, onde o paciente está com seu nível de consciência alterado e induz o paciente a elicitar a sua espiritualidade. O hipnoterapeuta realiza comandos que vão induzindo o paciente a se reconctar consigo mesmo, com seu interior e com a natureza e, assim, a compreender e superar o momento de angustia que está vivenciando.

Já o mindfulness é conceituado no artigo como uma "prática psicológica positiva budista" (Casula, 2018) que promove o profundo autocuidado. Além disso, é estar consciente e atento para os pensamentos, sensações e emoções, ajuda o individuo ao compreender e aceitar quem é e suas experiências de vida. $\mathrm{O}$ artigo aborda que essas duas práticas ajudam ao paciente a viver com foco no presente, deixando a negatividade e o passado para trás.

O artigo 03 foca na prática da terapia ocupacional, onde o processo terapêutico é focado em atividades que promovam qualidade de vida e assessorar ao paciente a viver ativamente. Assim, a terapia ocupacional possui algumas abordagens presentes no texto que podem ser relacionadas com a espiritualidade e em alguns casos, práticas religiosas, como: "manutenção das atividades significativas para o doente e sua família"; "orientação e realização de medidas de conforto e controle de outros sintomas"; "criação de espaços de convivência e interação, pautados nas potencialidades dos sujeitos; apoio, escuta e orientação ao familiar e/ou cuidador" (Elmescany \& Barros, 2015). Focando na dimensão espiritual, procura identificar quais são as práticas que o paciente mais se identifica e que o mais aproxima de sua espiritualidade, favorecendo a realização de tal atividade baseada em seus valores e crenças.

No artigo 04, a esperança proporcionada através da espiritualidade aparece como bússola para a procura do sentido da vida, diminuição do medo da morte, manutenção do desejo de prolongar a vida e de planejar atividades futuras. Além disso, este artigo (bem como o artigo 01) citou brevemente a logoterapia. A logoterapia, também conhecida como "Psicoterapia do Sentido da Vida", foi desenvolvida por Viktor Frankl e possui, sobretudo, um cunho humanista. Essa escola psicológica se propõe a compreender a existência através de vivências nomeadamente humanas e reconhece a dimensão espiritual do sujeito (Moreira \& Holanda, 2010). Já o artigo 05 apresenta uma teoria do desenvolvimento da espiritualidade, que pode ser amplamente utilizada para a compreensão da espiritualidade de pacientes jovem-adultos com câncer. A negligência com a dimensão espiritual propicia a negligência do cuidado paliativo como um todo, principalmente para os jovens adultos, uma vez que há pouco conhecimento sobre as necessidades espirituais dessa população por falta de estudos e pesquisas. 
As técnicas e abordagens apresentadas nos cinco artigos analisados são pouco diretivas quanto a ações que o psicólogo e outros profissionais de saúde podem realizar para tratar das questões religiosas e espirituais com seus pacientes. Um artigo de Koenig (2004) apresenta algumas diretrizes aos médicos, que podem ser realizadas também pelas psicólogas, como: colher a história individual do paciente no que se refere à espiritualidade. Essa atitude é a primeira que deve ser realizada, pois oferecerá ao profissional informações sobre o que a relação do paciente com a R/E. A partir disso, o profissional pode consultar os serviços de capelão em contexto hospitalar para saber quais as melhores ações a realizar.

Além disso, o psicólogo Júlio Peres cita que existem escalas sistematizadas e validadas para abordar as questões da espiritualidade (Panzini \& Bandeira, 2007; Moreira-Almeida, Peres, Aloe, Lotufo Neto, \& Koenig, 2008; Marques, Sarriera, \& Dell'Aglio, 2009) e que podem ser utilizadas em cuidados paliativos, mas ele não recomenda orar junto com paciente, por exemplo, pois os estudos sobre essa abordagem ainda são insuficientes (Peres et al., 2007).

A integração das dimensões espirituais e religiosas dos pacientes com o trabalho do psicólogo "requer profissionalismo ético, alta qualidade de conhecimento e habilidades para alinhar as informações coletadas sobre as crenças e valores ao benefício do processo terapêutico" (Peres et al., 2007, p. 138). Todo profissional deve reconhecer seus limites a tratar do tema com seus pacientes, como por exemplo, não deve prescrever atividades religiosas/espirituais ao paciente se não for do interesse dele, nem orientar espiritualmente o paciente se não tiver tido treinamento e também não devem realizar qualquer ação que não seja centrada e dirigida ao paciente (Koenig, 2004).

\section{Considerações finais}

Este artigo abordou a temática da espiritualidade inserida no contexto de cuidados paliativos, tendo atingido o seu objetivo de analisar na literatura produções sobre as interfaces entre psicologia e espiritualidade/religiosidade no contexto de cuidados paliativos, através de uma revisão integrativa de literatura. O método proporcionou uma análise ampliada e holística do objeto, bem como a seleção e utilização de estudos de diferentes abordagens.

Os artigos analisados neste estudo demonstraram, de uma forma geral, que no contexto de cuidados paliativos a espiritualidade aparece como uma necessidade do paciente e de sua família e, dessa forma, é recomendável que faça parte do cuidado integral de pacientes com doenças terminais por possibilitar a manutenção da esperança e da fé, amenização de angustias e a ressignificação do sentido de vida e morte. Esses achados corroboram com os resultados encontrados nos estudos utilizados como referência, que afirmaram que a espiritualidade possui um lugar indispensável dentro da prática dos cuidados paliativos.

Foi encontrado que, apesar dos conceitos de espiritualidade serem distintos, eles se aproximam, se assemelham e se completam. No entanto, a pluralidade de definições pode ser um obstáculo para a compreensão dessa dimensão, principalmente por parte do psicólogo e de outros profissionais da área de saúde e, assim, prejudicar a sua prática e reconhecimento. É necessário também preencher a lacuna identificada sobre o aspecto da religiosidade, bem como a definição e sua expressão para esses pacientes.

Os artigos apresentaram algumas técnicas e abordagens que estão disponíveis e possibilitam o trabalho da espiritualidade do paciente. No entanto, esses manejos ainda são insuficientes, necessitando de mais estudos e pesquisas na área que identifiquem não só novas formas para a profissional de psicologia lidar e abordar a dimensão espiritual do paciente, bem como conhecer quais são as práticas que tem sido utilizadas por esses profissionais em atuação no contexto de cuidados paliativos. Foi identificado a escassez de práticas dos psicólogos frente à dimensão espiritual, evidenciando que existem poucas abordagens teóricas que possibilitem à profissional de psicologia lidar com esse aspecto do sujeito.

É um fato que, em geral, dentro do ambiente acadêmico nos cursos de psicologia não existe um espaço para a discussão e estudos sobre a espiritualidade, religiosidade e religião, ficando a critério e interesse do estudante buscar sozinho sobre esses conteúdos. 
Isso ratifica a existência de uma lacuna na prática do psicólogo diante da dimensão espiritual do outro, por não haver conhecimento ou treinamento prévio durante a formação.

Como consequência, percebe-se poucos estudos e pesquisas realizadas por e sobre a prática dos psicólogos relacionadas com a espiritualidade no contexto de cuidados paliativos. Todos os artigos explicitaram a necessidade de mais produções científicas que abordem a temática em questão para oferecer uma base prática mais solidificada para os profissionais de saúde, principalmente os psicólogos.

No que se refere às limitações do estudo, acreditamos que a pouca quantidade de artigos foi um limitador. Isso pode ter acontecido devido ao período de análise (2015-2018), pois quando a revisão foi realizada queríamos encontrar as pesquisas realizadas nos últimos anos. Além disso, selecionar apenas os artigos abertos pode ter impossibilitado o acesso a achados importantes. Nesse sentido, o presente artigo pretende-se exploratório e, com isso, evidenciou a escassez de literatura na área específica.

Portanto, é fundamental que os profissionais de psicologia realizem novos estudos e pesquisas para o fortalecimento desse campo. Conclui-se que o presente estudo foi de relevância para reconhecer a espiritualidade no contexto de cuidados paliativos, e também para assinalar e demonstrar a necessidade da realização de mais estudos que incluam a dimensão espiritual e a prática do psicólogo diante desse aspecto, que faz parte da integralidade do sujeito.

\section{Contribuições das autoras}

Aguiar BF participou da concepção, planejamento, delineamento, coleta e análise dos dados da pesquisa, interpretação dos resultados, redação do artigo científico. Silva JP participou da concepção, planejamento, interpretação dos resultados e redação do artigo científico.

\section{Conflitos de interesses}

Nenhum conflito financeiro, legal ou político envolvendo terceiros (governo, empresas e fundações privadas, etc.) foi declarado para nenhum aspecto do trabalho submetido (incluindo, mas não se limitando a subvenções e financiamentos, participação em conselho consultivo, desenho de estudo, preparação de manuscrito, análise estatística, etc.).

\section{Referências}

Almeida, A. M. (2009). Espiritualidade \& Saúde Mental: O desafio de reconhecer e integrar a espiritualidade no cuidado com nossos pacientes. Zen Review, 1-6. http://www. espiritualidades.com.br/Artigos/M_autores/MOREIRAALMEIDA_Alexander_tit_Espiritualidade_e_Saude_Mental. pdf

Alves, R. F., Melo, M., Andrade, S., \& Sousa, V. (2014). Knowledge and practices about palliative care for psychologists active at public hospitals. Psicologia, Saúde \& Doenças, 15(01), 77-95. https://doi.org/10.15309/14psd150108

Arrieira, I. C. O., Thoferhn, M. B., Schaefer, O. M., Fonseca, A. D., Kantorski, L. P., \& Cardoso, D. H. (2018). O sentido do cuidado espiritual na integralidade da atenção em cuidados paliativos. Revista Gaúcha de Enfermagem, 38(3):e58737. https://doi.org/10.1590/19831447.2017.03.58737

Barbosa, R. M. M., Ferreira, J. L. P., Melo, M. C. B., \& Costa, J. M. (2017). A espiritualidade como estratégia de enfrentamento para familiares de pacientes adultos em cuidados paliativos. Revista da SBPH, 20(1), 165182. http://pepsic.bvsalud.org/scielo.php?script=sci abstract\&pid=S1516-08582017000100010\&lng=pt\&nrm= iso

Benites, A. C., Neme, C. M. B., \& Santos, M. A. (2017). Significados da espiritualidade para pacientes com câncer em cuidados paliativos. Estudos de Psicologia, 34(2), 269-279. https://doi. org/10.1590/1982-02752017000200008

Borges, M. S., Santos, M. B. C., \& Pinheiro, T. G. (2015). Representações sociais sobre religião e espiritualidade. Revista Brasileira de Enfermagem, 68(4), 609-16. https://doi. org/10.1590/0034-7167.2015680406i

Cervelin, A. F., \& Kruse, M. H. L. (2014). Spirituality and religiosity in palliative care: Learning to govern. Escola Anna Nery - Revista de Enfermagem, 18(1),136-142. https://doi. org/10.5935/1414-8145.20140020

Elmescany, É. N. M., \& Barros, M. L. P. (2015). Espiritualidade e terapia ocupacional: Reflexões em cuidados paliativos. Revista do NUFEN, 7(2), 1-24. http:// pepsic.bvsalud.org/scielo.php?script=sci arttext\&pid=S2175-25912015000200002

Evangelista, C. B., Lopes, M. E. L., Costa, S. F. G., Abrão, F. M. S., Batista, P. S. S., \& Oliveira, R. C. (2016). Spirituality in patient care under palliative care: A study with nurses. Escola Anna Nery, 20(1), 176-182. https://doi. org/10.5935/1414-8145.20160023

Gomes, A. L. Z., \& Othero, M. B., (2016). Cuidados paliativos. Estudos Avançados, 30(88), 155-166. https://doi. org/10.1590/s0103-40142016.30880011 
Henning, M. C., \& Geronasso, T. D. (2009). A influência da religiosidade do cliente na saúde mental e na prática clínica da psicologia. Ágora: Revista De divulgação científica, 16(1), 171-176. https://www.periodicos.unc.br/index.php/ agora/article/view/20

Koenig, H. G. (2004). Religion, Spirituality, and Medicine: Research Findings and Implications for Clinical Practice. South Med J, 97(12), 1194-1200. https://doi.org/10.1097/01. SM].0000146489.21837.CE

Koenig, H. G. (2012). Religion, Spirituality, and Health: The Research and Clinical Implications. International Scholarly Research Notices, 2012:278730. https://doi. org/10.5402/2012/278730

Koenig, H. G., Hooten, E. G., Lindsay-Calkins, E., \& Meador, K. G. (2010). Spirituality in Medical School Curricula: Findings from a National Survey. The International Journal of Psychiatry in Medicine, 40(4), 391-398. https://doi. org/10.2190/PM.40.4.c

Koenig, H., Koenig, H. G., King, D., \& Carson, V. B. (2012). Handbook of Religion and Health. Oxford University Press.

Marques, L. F., Sarriera, J. C., \& Dell'Aglio, D. D. (2009). Adaptação e validação da Escala de Bem-estar Espiritual (EBE): Adaptation and validation of Spiritual Well-Being Scale (SWS). Avaliação Psicológica, 8(2), 179-186. http://pepsic. bvsalud.org/scielo.php?script=sci_abstract\&pid=S167704712009000200004\&lng=pt\&nrm=iso\&tlng=pt

Marques, S. M. S., Marques, S. S., \& Ribeiro, T. A. N. (2016). Religiosidade, espiritualidade, sentido existencial e saúde. Revista Científica Semana Acadêmica, 1(82). https://semanaacademica.org.br/artigo/religiosidadeespiritualidade-sentido-existencial-e-saude

Melo, C. F., Sampaio, I. S., Souza, D. L. A., \& Pinto, N. S. (2015). Correlação entre religiosidade, espiritualidade e qualidade de vida: Uma revisão de literatura. Estudos e Pesquisas em Psicologia, 15(2), 447-464. https://doi.org/10.12957/ epp.2015.17650

Mendes, K. D. S., Silveira, R. C. C. P., \& Galvão, C. M. (2008). Revisão integrativa: Método de pesquisa para a incorporação de evidências na saúde e na enfermagem. Texto \& Contexto - Enfermagem, 17(4), 758-764. https://doi.org/10.1590/ $\underline{\text { S0104-07072008000400018 }}$

Mistretta, E. G. (2017). Spirituality in young adults with end-stage cancer: A review of the literature and a call for research. Annals of Palliative Medicine, 6(3), 279-283. https://doi. org/10.21037/apm.2017.06.17
Moreira, N., \& Holanda, A. (2010). Logoterapia e o sentido do sofrimento: Convergências nas dimensões espiritual e religiosa. Psico-USF, 15(3), 345-356. https://doi. org/10.1590/S1413-82712010000300008

Moreira-Almeida, A., Lotufo Neto, F., \& Koenig, H. G. (2006). Religiousness and mental health: A review. Revista Brasileira de Psiquiatria, 28(3), 242-250. https://doi. org/10.1590/S1516-44462006005000006

Moreira-Almeida, A., Peres, M. F. P., Aloe, F., Lotufo Neto, F., \& Koenig, H. G. (2008). Portuguese version of Duke Religious Index: DUREL. Revista de Psiquiatria Clínica, 35(1), 31-32. https://doi.org/10.1590/S0101-60832008000100006

Nascimento, L. C., Santos, T. F. M., Oliveira, F. C. S., Pan, R., Flória-Santos, M., \& Rocha, S. M. M. (2013). Espiritualidade e religiosidade na perspectiva de enfermeiros. Texto \& Contexto - Enfermagem, 22(1), 52-60. https://doi. org/10.1590/S0104-07072013000100007

Oliveira, M. R., \& Junges, J. R. (2012). Saúde mental e espiritualidade/religiosidade: A visão de psicólogos. Estudos de Psicologia (Natal), 17(3), 469-476. https://doi. org/10.1590/S1413-294X2012000300016

Panzini, R. G., \& Bandeira, D. R. (2007). Coping (enfrentamento) religioso/espiritual. Archives of Clinical Psychiatry, 34(1), 126-135. http://www.scielo.br/pdf/rpc/v34s1/a16v34s1.pdf

Peres, J. F. P., Simão, M. J. P., \& Nasello, A. G. (2007). Espiritualidade, religiosidade e psicoterapia. Archives of Clinical Psychiatry, 34(Suppl 1), 136-145. https://doi. org/10.1590/S0101-60832007000700017

Peres, M. F. P., Arantes, A. C. L. Q., Lessa, P. S., \& Caous, C. A. (2007). A importância da integração da espiritualidade e da religiosidade no manejo da dor e dos cuidados paliativos. Revista de Psiquiatria Clínica, 34(Supl 1), 82-87. https://doi.org/10.1590/S0101-60832007000700016

Peters, J. L. (2015). A história das religiões no contexto da história cultural. FDC, 1(1), 87-104. https://periodicos.ufjf.br/index. php/facesdeclio/article/view/26418

Pompeo, D. A., Rossi, L. A., \& Galvão, C. M. (2009). Revisão integrativa: Etapa inicial do processo de validação de diagnóstico de enfermagem. Acta Paulista de Enfermagem, 22(4), 434-438. https://doi.org/10.1590/S010321002009000400014 